\title{
Utilizing next-generation sequencing in the management of multiple myeloma
}

\section{Marta Lionetti \& Antonino Neri}

To cite this article: Marta Lionetti \& Antonino Neri (2017): Utilizing next-generation sequencing in the management of multiple myeloma, Expert Review of Molecular Diagnostics, DOI:

10.1080/14737159.2017.1332996

To link to this article: http://dx.doi.org/10.1080/14737159.2017.1332996

Accepted author version posted online: 19 May 2017.

Submit your article to this journal $\pi$

View related articles $\sqsubset$

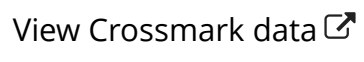


Publisher: Taylor \& Francis

Journal: Expert Review of Molecular Diagnostics

DOI: $10.1080 / 14737159.2017 .1332996$

Review

\section{Utilizing next-generation sequencing in the management of multiple myeloma}

Marta Lionetti ${ }^{1,2}$, Antonino Neri ${ }^{1,2}$

${ }^{1}$ Department of Oncology and Hemato-oncology, Università degli Studi di Milano; ${ }^{2}$ Hematology, Fondazione IRCCS Cà Granda Ospedale Maggiore Policlinico, Milano, Italy.

\section{Corresponding authors:}

Marta Lionetti, PhD

Antonino Neri, MD, PhD

Dept. of Oncology and Hemato-oncology

Università degli Studi di Milano

via Santa Sofia, 9, 20122 Milano, Italy

Phone: +39 02-50320420

Fax: +39 02-50320403

e-mail: marta.lionetti@unimi.it

antonino.neri@unimi.it 


\section{ABSTRACT}

Introduction: Multiple myeloma ( $\mathrm{MM})$ is a bone marrow plasma cell malignancy characterized by wide clinical presentation and heterogeneous genetic background. Despite the recent advances in patient outcome, new markers are needed for improving risk prediction and choice of a more appropriate therapy. In this perspective, the genetic makeup of MM cells is being better characterized by means of next-generation sequencing (NGS) technologies.

Areas covered: The authors discuss how the application of NGS has improved our knowledge of MM biology by discovering its mutational landscape, identifying the operating mutational processes, and revealing the clonal composition of tumors and the dynamics of its evolution; and how this can have important clinical implications in terms of prognostication, therapeutic choices, and response assessment. Finally, the authors provide a quick outlook of future applications of these technologies that could help in the management of the disease in the next years.

Expert commentary: The clinical exploitation of NGS-based characterization of MM patients has as its ultimate goal the precision medicine. Considerable obstacles to its implementation in myeloma management exist; therefore, the concerted effort of all involved stakeholders is mandatory to ensure that it will become a reality in routine clinical practice in the next future.

Keywords: next-generation sequencing; plasma cell dyscrasias; precision medicine; prognostication; response assessment; somatic mutations; tumor evolution. 


\section{Introducing multiple myeloma}

Multiple myeloma $(\mathrm{MM})$ is a malignant proliferation of antibody-secreting bone marrow (BM) plasma cells (PCs) that accounts for $10 \%$ of all hematological malignancies with an incidence in Western countries of about 3-5 per 100,000. PC dyscrasias show a wide clinical presentation spanning from the presumed pre-malignant condition of monoclonal gammopathy of undetermined significance (MGUS) to smoldering MM (SMM), symptomatic MM, and extra-medullary MM or plasma cell leukemia $(P C L)$ [1]. PCL can be primary $(p P C L)$ if originating de novo without any previous experience of $\mathrm{MM}$, or secondary ( $\mathrm{SPCL}$ ) when arising from a preexisting myeloma tumor that eventually progressed to the leukemic phase $[2,3,4]$.

Similarly to the clinical course, also the genetic background of the disease is highly heterogeneous as featured by a deep genetic instability, at both karyotypic and mutational level. Karyotypic instability involves structural and numerical aberrations [1,5]. About half of MM patients are hyperdiploid, carrying non-random trisomies of odd chromosomes and small incidence of chromosomal translocations at the immunoglobulin heavy chain (IGH) locus (14q32). The other cases are non-hyperdiploid and often display the constitutive activation of CCND1 (11q13), CCND3 (6p21), MAF (16q23), MAFB (20q11), or FGFR3/MMSET (4p16.3) genes, resulting from IGH translocations. Hyperdiploidy typically implies a better outcome, while $t(4 ; 14)$ and $t(14 ; 16)$ translocations are related to a dismal prognosis. In addition, imbalances of specific chromosomal portions including 17p13,1p,16q, 14q losses and 1q gains are negative prognostic factors [1, 6,7 , 8, 9]. Despite the recent advent of more efficient drugs (new generation proteasome inhibitors and immunomodulatory agents, and monoclonal antibodies) that have improved patient outcome and quality of life, most of patients relapse. In addition, in high-risk cases the disease is characterized by poor prognosis and short survival, not always predicted by the actually recommended risk stratification models, which combine biologic criteria (serum beta-2 microglobulin, albumin and lactate dehydrogenase levels) and cytogenetic abnormalities [17p13 deletion, $t(4 ; 14)$, and $t(14 ; 16)]$ $[10,11]$. Thus, the definition of new markers is made urgent, for not only improving risk prediction, but also to guide towards a more appropriate therapeutic strategy, taking full advantage of the available additional novel treatment options. This scenario has definitely represented an important 
rationale behind the efforts to characterize the genetic landscape of MM cells made in the recent years by means of next generation sequencing technologies (NGS). Here, we describe how NGS has improved our knowledge of MM biology by discovering its mutational landscape, identifying the mutational processes operative in the disease, and revealing its complex clonal composition and evolution. Furthermore, we discuss the clinical implications of NGS in terms of prognostication, therapeutic choices, and response assessment in the disease. Finally, we provide a quick outlook of future applications of these technologies that could contribute to further dissect the biology of MM cells and help in the management of the disease in the next future.

\section{Whole-genome and whole-exome sequencing findings in $M M$}

\section{$2.1 \quad$ Mutational landscape}

Three main studies analyzed large cohorts of MM patients by means of whole genome sequencing (WGS) and whole exome sequencing (WES). In particular, Lohr and colleagues analyzed a total of 203 tumor-normal pairs collected from 93 untreated and 101 previously treated patients, 177 of whom by WES and 26 by WGS [12, 13]; Bolli et al. profiled by WES 67 patients (52 at diagnosis and 15 treated), 15 of whom serially analyzed [14]; Walker and colleagues performed WES in 463 newly diagnosed MM patients enrolled in the UK National Cancer Research Institute Myeloma XI trial [15].

The emerging scenario from these studies indicates only a few genes found mutated at a significant frequency in each of the analyzed cohorts, some of which highlighting key pathways commonly deregulated (Figure 1).

KRAS and NRAS kinases are by far the most frequently mutated genes in MM (on average in $23 \%$ and $21 \%$ of patients, respectively). Along with BRAF (Figure 2), mutated to a lesser extent ( $8.7 \%$ of cases) but still at high statistical significance, they contribute to make the mitogen-activated protein kinase (MAPK) pathway the main target for somatic mutation in MM, as confirmed by a targeted resequencing analysis on a large representative panel of patients at different stages of PC dyscrasia [16]. Notably, the classic V600E variant is carried by less than half of the BRAF-mutated patients, unlike what observed in other tumor types, i.e. melanoma, colorectal cancer, and papillary 
thyroid carcinoma. Mutations in the RAS/MAPK pathway are reported to have no impact on survival [15].

The involvement in MM of DIS3 and FAM46C, which are two of the most recurrently mutated genes in the disease (average mutation frequency of $6.8 \%$ and $9.1 \%$, respectively) (Figure 3 ), was totally unexpected prior to the application of massively parallel sequencing. DIS3 belongs to the human exosome complex; it is endowed with both exo- and endonucleolytic activities and regulates the processing and amount of all RNA species [17]. Although the significance of its alteration in the disease still needs to be further elucidated, it is considered a potential tumor suppressor in MM based on several findings: the loss of enzymatic activity caused by the MMassociated DIS3 mutations that have been functionally characterized [18]; the loss of heterozigosity often involving DIS3 due to simultaneous gene mutations and chromosome 13 deletion [19]; and the reported enhanced translation of crucial oncogenes following DIS3 inactivation [20]. Similar to DIS3, FAM46C is an RNA-binding protein whose exact functions remain to be characterized, but potentially regulating gene expression [21]. FAM46C too has a putative tumor suppressor role in $\mathrm{MM}$, as suggested by the deletion of its genomic locus at $1 \mathrm{p} 12$, occurring in approximately $20 \%$ of $\mathrm{MM}$ patients [22], and the predominantly inactivating nature of MMassociated gene mutations [23]. Overall, the significantly recurrent alteration of DIS3 and FAM46C suggests a role of translational control processes in the pathogenesis of MM [12].

TP53 is mutated at a significant recurrence rate in all three studies (on average $8.3 \%$ ), although the mutational frequencies observed in each series are significantly different, and higher in series where advanced disease was over-represented [13, 14] (Figure 4). In fact, as highlighted by an NGS-based analysis in a representative cohort including patients with newly diagnosed MM and more advanced stages of PC dyscrasia, TP53 gene mutations are generally rare in MM at onset, and conversely constitute a marker of progression, analogously to 17p-deletion [24]. The incapacity to trigger an efficient apoptotic response to DNA damage (as defined by combining TP53 alterations with mutations observed in ATR, ATM, ZFHX4 or NCKAP5 genes, also involved in the DNA-repair pathway) represented the most significant prognostic mutational marker in the Myeloma XI trial [15]. 
Based on the three studies considered above, the process of $\mathrm{B}$ cell differentiation resulted frequently targeted by mutational events. Particularly affected were the genes encoding the SP140 nuclear body protein, i.e. the interferon-inducible lymphoid-restricted homolg of SP100; the lymphotoxin beta LTB, that is involved in physiological lymphoid development and in the induction of the inflammatory response system; and the transcription factors PRDM1 and IRF4, essential to plasma cell differentiation. Average mutation rates of SP140, LTB, PRDM1 and IRF4 genes are of $3.6 \%, 2.8 \%, 1.6 \%$ and $1.5 \%$, respectively. Notably, mutations affecting IRF4, a target of the IMID drugs whose inhibition is toxic to MM cell lines [25], are preferentially represented by the K123R variant and have been reported to have a positive impact on survival in the Myeloma XI trial [15].

EGR1 is a transcriptional regulator inducing apoptosis in MM cells, where ts knockdown enhanced resistance to Bortezomib [26]. Similarly to IRF4, EGR1 is an IMID target whose mutations, detected in $4.3 \%$ of $\mathrm{MM}$ patients and clustered at the 5 end of the gene, were found correlated with good overall survival by Walker and colleagues [15].

The identification of mutations in multiple components of the NF-KB signalling (especially TRAF3, with an average mutation rate of $3.9 \%, C Y L D$, mutated in $2.6 \%$ of cases, and the aforementioned $L T B)$ further supported and extended the anticipated role of this pathway $[27,28]$ and widened our knowledge on the mechanisms of its activation in MM.

Among the genes found mutated in MM patients, some are involved in cell cycle regulation, in particular RB1 and CCND1, altered respectively in $1.3 \%$ and $2.7 \%$ of patients; this latter seems to be associated with a negative impact on survival [15].

\subsection{Mutational signatures}

Two main studies employed non-negative matrix factorization (NMF) to extract mutational signatures from WES data and shed light on the mutational processes contributing to the MM mutational landscape $[14,29]$. NMF analyses identified at least two mutational signatures: the most frequent was called Signature A, it corresponds to signatures 1 and 5 in the paper of Alexandrov et al. [30] and is a quite general signature characterized by $C>T$ mutations in a CpG context, a process attributed to the spontaneous deamination of methylated cytosine and its conversion to 
thymine. In a small percentage of patients characterized by a high mutational load, a significant fraction of the mutational repertoire is ascribable to Signature $B$, a second signature that corresponds to Signature 2 in the paper of Alexandrov et al. [30]. This signature results from the aberrant activity of APOBEC (apolipoprotein B mRNA editing enzyme, catalytic polypeptide-like) family of DNA editing enzymes. Interestingly, APOBEC signature is enriched in MM patients harboring $t(14 ; 16)$ and $t(14 ; 20)$ translocations overexpressing respectively $M A F$ and $M A F B$ transcription factors, which likely control $A P O B E C 3 B$ and $A P O B E C 4$ expression [29]. Recently, also a novel signature characterized by small peaks at "C>T GCA" and "C>T GCC" motifs has been reported as significantly enriched in acquired mutations in two MAF-translocated patients [31].

While aberrant APOBEC activity has been associated with a higher load of mutations overall, sometimes it results in areas of localized hypermutation, a phenomenon called kataegis, that it is often found at the boundaries of somatic genomic rearrangements. Another instance of aberrant somatic hypermutation is mediated by off-target activity of AID, a deaminase responsible for somatic hypermutation during normal differentiation of germinal center B-lymphocytes undergoing antigen selection in adaptive immunity. Instances of mutations within AID recognition motifs have been found in the partner oncogenes of IGH translocations [14, 29], and their role in myeloma pathogenesis remains to be elucidated.

Finally, the examination of mutational signatures over time in a small fraction of patients indicated that the relative contribution of diverse mutational processes involved in the generation of the mutational repertory in MM may change over time [14].

\subsection{Clonal heterogeneity}

Massively parallel-sequencing studies $[13,14]$ corroborated the intratumor heterogeneity of MM anticipated by several phenotypic, molecular and clinical findings [32]. Beyond the major tumor clone, indeed, nearly all patients had evidence of clonal heterogeneity, carrying variants occurring only in a portion of MM cells. The awareness that intra-clonal heterogeneity is an hallmark of MM 
biology has modified our way of approaching cancer, now considered as a composite mixture of clones and not as a linear evolving disease [33].

The subclonal nature of not only supposed passenger mutations but also supposed driver mutations, such as those in KRAS, NRAS, and BRAF genes, indicates that these latter might occur late in the evolution of the disease, reflecting a dynamic mutational process. In several cases, multiple mutations in oncogenes with redundant functions (i.e. KRAS, NRAS and BRAF genes) were seen in the same tumor sample, although generally these mutations did not co-occur clonally. In this regard, a single-cell analysis of multiple mutations in the RAS/MAPK pathway within the same MM tumor revealed the occurrence of convergent evolution, consisting in the acquisition of redundant advantageous mutations by distinct clones, which subsequently evolve independently [34]. As discussed later in the text, the subclonal nature of variants affecting driver genes and multiple coexisting substitutions in the same pathway has important therapeutic implications.

WES and WGS on a small cohort of patients at various phases of PC dyscrasia (four MGUS, four high-risk SMM, 26 MM and two PCL samples, including four MM cases who evolved from SMM) indicated that the genetic complexity increases with disease stage [35]. However, intraclonal heterogeneity appears to be a premature event in myelomagenesis, already present before the occurrence of clinical symptoms, suggesting that immortalized plasma cells diverge very early in their evolution. Intra-tumor diversity is critical for disease progression, being the essential substrate for underlying Darwinian-like tumor evolution, as discussed further in the following paragraph.

\subsection{Clonal evolution}

The application of massive parallel sequencing revealed a previously unsuspected model of genomic evolution in MM, that diverges from the traditional dogma of genomic aberrations linearly accumulating over time. It rather suggests a Darwinian branching model of tumor evolution, with several clonal progenitors present at diagnosis whose dominance alternates over time under selective pressures exerted by microenvironment or therapy [36, 37]. Along with branching evolutionary pathways, responsible for the presence of one or more new clones (likely evolving 
from ancestral minor clones with newly acquired mutations) and the decreased frequency or loss of others, additional patterns of tumor evolution observed in serially analyzed MM patients include: clonal stability (i.e. no change in the clonal composition), linear evolution (i.e. the emergence of a new subclone), and differential clonal response. This latter pattern consists in an alteration of the relative proportions of each subclone which may be related to their random drift in the course of time; different response to therapy among subclones; or subclone expansion due to selective advantage [14].

To gain further information about the pattern of evolution underlying relapse in $\mathrm{MM}$, a recent longitudinal study investigated by WES, gene expression profiling and high-resolution copy number arrays 33 patients enrolled in Total Therapy protocols with the aim to define specific drivers of relapse in the precise context of a standard treatment [31]. Notably, the acquisition/expansion of mutations in known MM driver genes (NRAS, KRAS, BRAF) and the biallelic inactivation of tumor suppressor genes, especially TP53, proved to be critical events driving relapse through the escape form treatment-induced apoptosis and increased proliferation, leading to drug resistance and tumor progression. In particular, biallelic events resulting in complete inactivation of TP53 defined the group with the worst outcome after relapse. The excess of biallelic events and copy number changes from presentation to relapse in high-risk cases were consistent with increased genomic instability associated with these patients. The Authors proposed a model combining "Big Bang" and Darwinian type of evolution, in which "Big Bang" dynamics lead to the early establishment of intratumor heterogeneity, followed by Darwinian-type evolution, in which different subclones acquire additional aberrations and compete with each other and normal hematopoiesis to access to an appropriate bone marrow niche. In this scenario, treatment generates a significant evolution bottleneck, which eradicates some subclones but may simultaneously select for clones with strong driver events that increase proliferation and resistance to apoptosis.

Finally, it is worth reporting that a very recent analysis of the distributions of variant allele frequencies determined from WES data of 463 patients from the UK Myeloma XI trial indicated that nearly $20 \%$ of MM tumors at diagnosis are under neutral evolutionary dynamics, consistent with 
the lack of natural selection during malignant growth [38] (Johnson et al., 16th International Myeloma Workshop, abstract \# 508). Neutral evolution seemed to be associated with $\operatorname{lgH}$ translocations, gain of $1 \mathrm{q} 21$, and reduced responsiveness to microenvironment-modulating IMiDtherapy. Further studies may help to confirm and extend these data.

\section{5. $\quad p P C L$}

PPCL is an infrequent, aggressive form of PC dyscrasia and may constitute an effective model for high-risk MM. A recent WES study investigated the mutational profiles of 12 PPCL patients enrolled in a multicenter prospective GIMEMA Phase II trial (www.clinicaltrials.gov as \#RV-PCL-PI-350, www.clinicaltrialsregister.eu as \#EudraCT $\mathrm{N}^{\circ}$ 2008-003246-28) [39]. Few genes were found recurrently mutated in two or more samples, confirming a substantial heterogeneity of mutational patterns, as observed in intramedullary disease. Concerning the genes found recurrently mutated in MM, mutations in KRAS and NRAS were three-fold less frequent, while those in DIS3 and TP53 were till to two-fold more recurrent in PPCL than in MM, and this was perhaps at least partly related to different representativeness of cytogenetic abnormalities in PPCL and MM series. Globally, extracellular matrix (ECM) components, the cadherin/Wnt signaling pathway and cell cycle checkpoint were found enriched in mutational events. Also based on gene expression data, the Authors speculated that alterations of ECM-receptor interaction and of cadherin/Wnt signaling pathway may be strongly involved in the mechanisms of extramedullary spread associated with pPCL. On the other hand, the recurrent involvement of DNA damage response genes [specifically TP53, ATM, ATR and other downstream genes associated with DNA repair activity (CHEK2, $C D C 25 A, C D K N 1 A, B R C A 1)]$ was suggestive of a potential primitive pathogenetic role of impaired DNA repair functions in $\mathrm{pPCL}$, unrelated to therapy [39]. Investigations in larger collections are needed to further dissect the molecular mechanisms responsible for this aggressive form of PC dyscrasia.

\section{Clinical implications of NGS-derived data}


The information made available by the application of NGS technologies in MM has potential relevance in the clinical setting at various levels, including prognostic stratification, therapeutic choices and response assessment.

\subsection{Prognostication}

To date, the potential of NGS in MM prognostication has not yet been fully exploited. In fact, the prognostic significance of the mutational spectrum characterizing $\mathrm{MM}$ has been assayed virtually only by Walker and colleagues within the clinical trial Myeloma XI, comparing a standard chemotherapy regimen of cyclophosphamide, dexamethasone plus thalidomide with a newer regimen of cyclophosphamide, dexamethasone plus lenalidomide [15]. In the context of this study, while mutations in MAPK and NF-kB pathways, as anticipated above, are prognostically neutral, variants in CCND1 and DNA repair pathway correlate with poor survival, and those in IRF4 and EGR1 appear to be associated with better prognosis. Notably, some of these new prognostic factors (including mutations in TP53, ZFHX4, CCND1, ATM and ATR) were combined with adverse cytogenetic abnormalities, such as del(17p), $\mathrm{t}(4 ; 14), \operatorname{amp}(1 \mathrm{q})$ and $M Y C$ translocations, and the International Staging System (ISS) to generate a risk score able to better identify high-risk patients experiencing relapse and premature death [15]. Although an external validation of this predictive tool is required, the Authors envisaged an easy incorporation of the detection of these molecular markers in a diagnostic test that could be developed in the everyday practice.

Beyond mutations affecting single genes, the prognostic power of patients' global mutational load is uncertain. Indeed, while Bolli et al. reported a positive correlation between the amount of variants and the extent of the risk of relapse and death [14], Weinhold and colleagues found no association between the mutational load and GEP70 risk status either at presentation or relapse, after adjustment for the UAMS (University of Arkansas for Medical Sciences) molecular calssification $[31,40]$.

\subsection{Therapy}

Concerning the therapeutic implications of the application of NGS-based technologies in MM, it is conceivable that the definition of MM mutational landscape may help towards the identification of 
specific treatments from which patients may particularly benefit, or otherwise to be avoided. This could be the case of patients carrying mutations associated with a clinical response to specific therapies or representing druggable targets. NRAS, IRF4 and EGR1 are examples of genes that, when mutated, seemed to be associated with a different response to treatments currently used in myeloma. In particular, an association was reported between the reduced sensitivity to bortezomib and the presence of NRAS mutations at relapse, having a negative impact on both response rate and time to progression [41], whereas IRF4 and EGR1 mutations correlated with better outcome with immunomodulatory agent therapy [15]. Another important finding of more general nature but with potential implications on therapeutic choices has recently emerged from the aforementioned longitudinal WES study by Weinhold and colleagues on MM patients relapsed after treatment with high dose therapy [31]. Indeed, the identification of the bi-allelic loss of tumor-suppressor genes, such as TP53, as a crucial mechanism allowing MM cells to evade treatment-induced apoptosis with the acquisition of proliferative advantage suggests the employment of alternate therapies during the post-induction phase, especially treatments inducing cell death via tumor-suppressor independent pathways.

Even more appealing is the perspective of directing therapy specifically to driver mutations, which are known to provide a fitness advantage to MM cells, by means of agents selectively inhibiting the mutated or activated oncogene. Notably, the most recent and comprehensive WES study in MM overall detected potentially druggable mutations in 309 target genes and involving the $53 \%$ of patients, suggesting an even broader applicability of targeted therapies in MM in the coming years (Table 1) [15]. Single agents targeting specific mutations are not currently approved for routine clinical use in MM, but several of such molecules are in clinical testing, and a few experiences concerning their successful employment in MM patients have begun to be reported, for example for the V600E-mutated BRAF inhibitor Vemurafenib [42, 43] and the MEK inhibitor trametinib [44]. Other actionable mutations include those affecting FGFR3, CCND1, and the pathway of NF-kB [27, 28], just to name a few. Although tailored therapies are promising, some biological aspects of the disease that may limit severely their effectiveness must be taken into consideration. Concerning the inhibition of MAPK pathway, for instance, Lohr and colleagues [13] 
reported a paradoxical increase in $\mathrm{pERK}$ and in cell proliferation in $B R A F$-wild type $\mathrm{MM}$ cell lines treated with Vemurafenib, as already observed in melanoma [45]. Notably, MAPK pathway activation was even more pronounced in the presence of KRAS or NRAS mutations. Translated in a clinical setting, these data cast doubts on the potential benefits of the use of BRAF inhibitors, especially if used as monotherapy, in MM patients harboring subclonal BRAF mutations or coexistent BRAF and RAS mutations in different cells of the same tumor. Under such circumstances, BRAF inhibitors may lead to paradoxical expansion of, respectively, BRAF-wild-type and RASmutated subclones and hence to clinical progression. The latter scenario was recently described in one instructive case, where a proteasome inhibitor-based therapy (bortezomib/dexamethasone) was used to overcome resistance to vemurafenib [46].

Furthermore, clonal heterogeneity might represent a major obstacle to the success of targeted therapies pinpointed to one genetic lesion. Indeed treatment directed against a mutation carried only by a subset of tumor cells is expected to be effective exclusively on that subclone, with a consequent restricted clinical benefit, which makes at least necessary to resort to a combination of different types of treatment targeting distinct subclones.

On the other hand, even genes mutated in most of the tumor population do not necessarily have an important biological impact. Targeted treatment based solely on DNA sequencing data, in fact, could be ineffective if that mutated gene is not expressed. Indeed, a recent RNA-seq analysis of a subset of patients who had previously undergone WES showed that most of the reported mutated genes have very low or undetectable expression [47].

\subsection{Response assessment}

NGS could also meet another strong need that is emerging in the management of MM patients, i.e. the determination of minimal residual disease (MRD) $[48,49]$. While achievement of a morphological and serological complete remission was rare in the past, novel treatments have resulted in an increased occurrence of these events so that nearly all patients show a response to treatment and most achieve a complete response (CR) [50]. However, the increased effectiveness of treatment approaches in the past decade has not been accompanied by a better definition of 
$\mathrm{CR}$, which conversely remains based on conventional serological and morphologic techniques, with the result that most patients achieving CR ultimately relapse and die. This scenario is mainly attributable to the persistence of MRD undetected by conventional response assessment methods [49]. Newer and more sensitive techniques to detect MRD in BM aspirates include multiparameter flow-cytometry (MFC), allele-specific oligonucleotide polymerase chain reaction (ASO-PCR) and NGS. Although currently none of these techniques fully satisfies all the characteristics that the ideal MRD test should possess (i.e., high applicability, high sensitivity and specificity good feasibility, easy accessibility, requirement for a limited sample, reproducibility, and proven clinical outcome), NGS fulfills most of them. In particular, MRD assessment by NGS is mainly, although not exclusively, based on the LymphoSIGHT platform (Adaptive Biotechnologies), which consists in genomic DNA amplification by multiplexed PCR assays using consensus primer sets allowing the recognition of all known alleles of the germline IGH and IGK sequences, followed by sequencing and identification of cancer-associated clonotypes with a minimum frequency of $5 \%$ [51]. The tumor-derived sequences detected in the samples at diagnosis are used as targets in follow-up samples to assess the presence of MRD, defined as the number of cancer-derived molecules per one million cell equivalents. Compared with ASO-PCR, NGS-based MRD assessment proved to be at least comparable in terms of sensitivity [52], and recent studies in chronic lymphocytic leukemia and acute lymphoblastic leukemia indicated that a sensitivity of one in 1,000,000 is achievable by NGS when higher amounts of DNA are used [53, 54]. Furthermore, contrary to ASO-PCR, NGSbased monitoring of MRD does require neither use of patient-specific primers, nor creation of a standard curve for quantitation. This latter and other limitations of quantitative ASO-PCR could be overcome, at the same performance level, by droplet digital PCR, especially in terms of reduced labor intensiveness and easier data interpretation, although its potential advantages and predictive value need to be further studied in the context of prospective clinical trials [55]. On the other hand, compared with NGS, MFC has the advantages of requiring less time to generate results, of being virtually applicable to all patients, and of not requiring a baseline sample. However, although a sensitivity higher than $10^{-5}$ is achievable by means of the new MFC instruments, up to $2 \times 10^{6}$ cells are required. In addition, further efforts are needed to conform the flow-based approaches and limit 
the subjectivity linked to individual interpretations. Finally, the need to work on fresh samples makes the transfer of MFC in clinical settings more complex. Nevertheless, NGS too has limitations: besides that fact that it can be limited in its ability to capture a clonotype in all patients in spite of adequate samples, there are still several issues with quantification, among which the calibration and correction approaches used to determine total leukocytes, B-lineage cell numbers, and reproducibility of quantification (in particular considering that it depends on DNA quantity and sequencing depth) [56]. Furthermore, additional checks are required to determine whether a negative result is attributable to sample quality (e.g., morphology and/or flow cytometry).

The clinical significance of sequencing-based MRD assessment in MM patients was recently reported in two studies by the same group $[51,57]$. In particular, the most recent one adopted an in-house deep-sequencing method using the standardized primers developed by the Biomed-2 concerted action to amplify all IGH or IGK sequences in a patient sample [58]. Differently from the proprietary multiplex PCR entailed by the LymphoSIGHT method, to be performed at centralized laboratories, this approach can be implemented in any laboratory with NGS capability, thus shortening turn-around time, and can be fully automated, and hence easily standardized reducing inter-lab variation [57]. Latest recommendations of the International Myeloma Working Group (IMWG) (2016) strongly encouraged the inclusion of next-generation sequencing and nextgeneration flow in prospective trials to find out the pros and cons of the single approaches, and the sensitivity required in different clinical settings. Notably, to harmonize the definition of MRD status across studies, the IMWG revised the response criteria for myeloma and included MRD negativity as the highest degree of response to treatment [59]; in particular, sequencing MRD-negativity was defined as absence of clonal PCs by NGS on BM aspirate.

\section{Expert commentary}

The exploitation of NGS-enabled mutational characterization of cancer patients in therapeutic field has as its ultimate goal the implementation of precision medicine, i.e. the administration of personalized treatment based on the mutational repertoire of each tumor. This process is often referred to as matching the right drug to the right patient in the right dose at the right time. A major 
obstacle to its implementation, however, is the inadequacy of many current clinical trial designs. Such inadequacy is related to the fact that targeted approaches rely on increasing amounts of therapeutic agents, whose potential combinations and conceivable sequential administration options are even exponentially more numerous. Furthermore, myeloma, like most cancers, is not a molecularly homogeneous entity, but rather presents several actionable mutations and does not nicely segregate into genomically-defined subtypes that could be used to stratify treatment arms [60]. In other words, the similarity between patients and the homogeneity between therapy groups required for classical clinical trials seem irreconcilable with the molecular intra- and inter-tumor diversity and different therapeutic options that constitute the basis of personalized medicine. The resulting combinatorial complexity makes it necessary to extend the conventional "basket" and "umbrella" approaches developed to study molecularly targeted therapies [61] and to resort at least to adaptive designs enabling an ongoing modification of the clinical trial based on the knowledge acquired through the trial itself [62]. Even better, precision medicine would require a kind of clinical trial focusing on single, not average, treatment responses, and probing the myriad factors that shape an individual's response to a specific therapy [63]. Such a personalization of clinical trials might rely on the implementation of oncological $\mathrm{N}$-of-1 trials (which should all be evenly carried out and whose results should be aggregated), although there is skepticism towards this approach in part of the oncology community, including regulatory agencies, researchers and clinicians [64].

Beyond the paradigm shift in clinical trial design needed to fit studies able to effectively assessing targeted agents into the regulatory drug approval pathway, another obstacle to the realization of precision oncology is the amount of data that must be taken into account and integrated, including high-quality NGS data, histopathological and clinical findings. The impossibility of processing and interpretation of such a data volume in a meaningful and timely manner by clinicians raises the need for machine-learning approaches intended to assist oncologists responsible of taking decisions about the treatment of single patients. IBM's Watson Oncology is one of these computing self-learning algorithms currently being tested at some institutions $[65,66]$. 
In our opinion, those just discussed are the main considerations to be made when thinking about a future pervasive application of NGS-enabled tailored medicine in MM, but certainly many others exist. After all, precision oncology represents an epochal revolution in patients' management, and therefore it is conceivable that it involves substantial changes (at both cultural and practical levels) in the way we operate in order to cure cancer, that surely will need a long time to be realized. The concerted effort of all stakeholders involved in the development of precision oncology (researchers, clinicians, regulatory agencies, governments) is now mandatory to ensure that in the future it will become a reality in routine clinical practice.

\section{Five-year view}

Considering that the continuous improvement of sequencing technologies will most likely reduce costs and processing times, in the near future NGS might really represent a viable option for routine clinical practice. In this perspective, clinical-grade approaches to analyze the genomic repertoire of $\mathrm{MM}$ patients allowing the identification of biomarkers with clinical relevance have begun to be developed and certainly will continue to be implemented. In this regard, Kortum and colleagues were among the first to demonstrate the practical use of a custom MM-specific gene panel for targeted sequencing as a simple, inexpensive and rapid strategy to characterize the mutational profile of MM. Specifically, they initially constructed a panel of 47 genes $\left(\mathrm{M}^{3} \mathrm{P}\right)$ [67] subsequently updated to a 77 gene version $\left(\mathrm{M}^{3} \mathrm{Pv} 2.0\right)$ [68], and more recently further integrated to include 88 frequently mutated or drug-resistance pathway MM genes, with a focus on genes relevant to $\mathrm{IMiD}$ and proteasome inhibitor interactions $\left(\mathrm{M}^{3} \mathrm{P}\right.$ 3 3.0) [69]. It is worth to point out also two recently published studies, both proposing a target-enrichment strategy followed by NGS for one-step identification of MM common genetic abnormalities. In particular, Bolli and colleagues developed and validated an approach to simplify all-in-one high-throughput analysis of gene mutations, DNA copy number changes and IGH translocations in MM independently from matched normal samples [70]. Similarly, the strategy proposed by Jimenez et al. allowed the identification of IGH translocations, $\mathrm{V}(\mathrm{D}) \mathrm{J}$ rearrangements and $\mathrm{IgH}$ isotype (usable as a target for evaluating MRD), and somatic mutations in a single run [71]. Although a substantial optimization of capture- 
based NGS panel and of data analysis is still required, these works represent the first appealing attempts of introducing NGS-based technologies during routine diagnostic workup for MM.

An even more widespread and fruitful application of NGS in routine clinical practice might be expected if the genetic analysis of circulating MM cells at single-cell level recently reported by Lohr et al should prove a viable approach [72]. In particular, by analyzing at single-cell resolution circulating tumor cells (CTCs) isolated from peripheral blood of $10 \mathrm{MM}$ patients, the Authors demonstrated that the genetic makeup of CTCs recapitulates MM in the bone marrow, even revealing mutations with greater sensitivity than bone marrow biopsies in some cases. Although larger studies will be required to establish its clinical utility, this approach would virtually allow to fully exploiting the potential of NGS, as it would make possible the extensive molecular monitoring of MM patients that actually does not occur due to the clinical impracticability of serial bone marrow sampling (involving an invasive and painful procedure). In particular, the ability to track the genetic evolution of MM could reveal the acquisition of mutations associated with the transition to overt MM in MGUS/SMM patients at risk of progression, thus warranting preventive interventions, as well as emerging drug resistance mutations in MM patients undergoing treatment, who might be directed towards early intervention with mechanism-driven therapies. Notably, recent evidence supports the feasibility of non-invasive mutational profiling of PC dyscrasia patients also from circulating cellfree tumor DNA [73, 74].

Continuing to speculate on the future evolution of NGS technology in MM, RNA-sequencing is an application of NGS still largely unexplored in the context of the disease [47, 75], and that reasonably will produce a substantial amount of new data in the near future.

In particular, RNA-seq could allow a deeper characterization of coding transcriptome than that obtained by means of microarrays, by providing information, as well as on gene expression levels, on differential splicing and isoform expression, mutational profiling and gene fusions. This could be particularly meaningful in MM, where disease-specific alternate splicing events of several genes have been reported $[76,77,78]$. Moreover, RNA-seq is the technology of choice for the discovery and genome-wide expression analysis of non-coding RNAs, whose involvement in MM is increasingly documented and that are being investigated as a therapeutic target $[79,80]$. 
Furthermore, recent large-scale epigenomic analyses of MM cells have highlighted a complex epigenomic landscape contributing to $\mathrm{MM}$ onset and prognosis and characterized by anomalies in DNA methylation and in post-translational modification of histone proteins [81]. However, epigenetic intra-tumor heterogeneity in MM is yet unexplored and, as regards DNA methylation, it might be analyzed by DNA methylation profiling through enhanced reduced representation bisulfite sequencing (ERRBS), as done by Pan and coworkers in diffuse large B cell lymphoma [82]. ERRBS, indeed, is an efficient and high-throughput, NGS-based technique used to analyze the genome-wide methylation profiles at single-nucleotide resolution, and provides a unique opportunity to quantify intra-tumor DNA methylation heterogeneity $(\mathrm{MH})$ since each sequenced read is derived from one individual tumor cell. Given the proved intra-tumor heterogeneity characterizing multiple myeloma at various levels (phenotypic, molecular, genetic and clinical), it is reasonable to expect the existence of epigenetic intra-tumor heterogeneity as well. The perspective of therapeutically targeting aberrant methylation and the prognostic value of $\mathrm{MH}$ in other cancer types encourage its assessment in MM.

\section{Key issues}

- $\mathrm{MM}$ is a malignant proliferation of antibody-secreting bone marrow plasma cells characterized by a wide clinical presentation and an extremely heterogeneous genetic background. Despite the recent improvement of patient outcome and quality of life thanks to new efficient drugs, the majority of patients experiences relapse, and the actually recommended risk stratification at diagnosis fails to identify a subgroup of high-risk cases.

- Recently, the genetic makeup of MM cells is being better characterized by means of NGS technologies, with the aim of defining new markers for improved risk prediction and to guide towards the most appropriate therapeutic strategy.

- The general scenario emerging form WGS/WES studies indicates a few genes found mutated at a significant frequency of occurrence, among which KRAS, NRAS, BRAF, TP53, DIS3 and FAM46C. Besides the MAPK pathway, that is the most recurrently mutated, the non-canonical NF-kB signalling is another key target of somatic mutation in MM. 
- Different processes, among which kataegis and aberrant somatic hypermutations, are involved in the generation of MM mutational repertory, whose relative contribution may vary in time.

- Nearly all patients had evidence of clonal heterogeneity. Even known driver mutations can be subclonal in some patients. An analysis at the single-cell level provided clear evidence of convergent evolution, consisting in the acquisition of redundant mutations conferring growth advantages by distinct clones. Although the genetic complexity increases with disease stage, clonal heterogeneity appears to be a premature event in MM pathogenesis and critical for disease progression.

- The application of massively parallel sequencing revealed, along with the traditional model of genomic aberrations linearly accumulated over time, a Darwinian branching model of tumor evolution, with several clonal progenitors present at diagnosis whose dominance alternates over time under selective pressures exerted by microenvironment or therapy.

- The information made available by the application of NGS technologies in MM is potentially reflected in the clinical setting at various levels, including i) prognostic stratification; ii) therapeutic choices; iii) response assessment.

i) Prognostication: a risk score with improved capacity to identify high-risk patients has been generated by combining some new mutation prognostic markers, adverse cytogenetic abnormalities and ISS [15].

ii) Therapeutic choices: in terms of therapeutic applications of NGS-derived data, the most appealing perspective is directing treatment specifically to driver mutations, by means of agents selectively inhibiting the mutated or activated oncogene. The most recent and comprehensive WES study in MM overall detected potentially druggable mutations in 309 target genes and involving $53 \%$ of patients [15]. Although tailored therapies are promising, some biological aspects of the disease that may limit severely their effectiveness must be taken into consideration, first of all clonal heterogeneity.

iii) In 2016, the IMWG revised the response criteria for myeloma and included MRD negativity as the highest degree of response to treatment. Sequencing-based MRD assessment fulfills 
almost all the characteristics that the ideal MRD test should possess, and its clinical significance is recently being reported $[51,57]$.

- Two major obstacles to the implementation of precision oncology in myeloma management are the inadequacy of many current clinical trial designs and the amount of data that must be taken into account and integrated, including high-quality NGS data, histopathological and clinical findings. The concerted effort of all stakeholders involved in the development of precision oncology (researchers, clinicians, regulatory agencies, governments) is mandatory to ensure that in the future it will become a reality in routine clinical practice.

- Some preliminary attempts aimed at introducing NGS-based technologies during routine diagnostic workup for MM have been made $[68,69,70,71]$, and certainly will continue to be implemented. 


\section{Acknowledgements}

The authors would like to thank Prof. N.Bolli for his critical reading of the manuscript.

\section{Funding}

This work was supported by the Associazione Italiana per la Ricerca sul Cancro under Grant IG 10136 and 16722 to A. Neri.

\section{Disclosures}

The authors have no relevant affiliations or financial involvement with any organization or entity with a financial interest in or financial conflict with the subject matter or materials discussed in the manuscript. This includes employment, consultancies, honoraria, stock ownership or options, expert testimony, grants or patents received or pending, or royalties. 


\section{References}

Reference annotations

* Of interest

** of considerable interest

1. Morgan GJ, Walker BA, Davies FE. The genetic architecture of multiple myeloma. Nature reviews Cancer. 2012;12:335-48.

2. Albarracin F, Fonseca R. Plasma cell leukemia. Blood reviews. 2011;25:107-12.

3. Musto P, Simeon V, Todoerti K, et al. Primary Plasma Cell Leukemia; Identity Card 2016. Current treatment options in oncology. 2016;17:19.

4. Neri A, Todoerti K, Lionetti M, et al. Primary plasma cell leukemia 2.0: advances in biology and clinical management. Expert review of hematology. 2016;9:1063-73.

5. Kuehl WM, Bergsagel PL. Multiple myeloma: evolving genetic events and host interactions. Nature reviews Cancer. 2002;2:175-87.

6. Anderson KC, Carrasco RD. Pathogenesis of myeloma. Annual review of pathology. 2011;6:249-74.

7. Corre J, Munshi N, Avet-Loiseau H. Genetics of multiple myeloma: another heterogeneity level? Blood. 2015;125:1870-6.

8. Manier S, Salem KZ, Park J, et al. Genomic complexity of multiple myeloma and its clinical implications. Nature reviews Clinical oncology. 2017;14:100-13.

9. Munshi NC, Avet-Loiseau H. Genomics in multiple myeloma. Clinical cancer research : an official journal of the American Association for Cancer Research. 2011;17:1234-42.

10. Chng WJ, Dispenzieri A, Chim CS, et al. IMWG consensus on risk stratification in multiple myeloma. Leukemia. 2014;28:269-77.

11. Palumbo A, Avet-Loiseau H, Oliva S, et al. Revised International Staging System for Multiple Myeloma: A Report From International Myeloma Working Group. Journal of clinical oncology : official journal of the American Society of Clinical Oncology. 2015;33:2863-9.

12. Chapman MA, Lawrence MS, Keats $\mathrm{JJ}$, et al. Initial genome sequencing and analysis of multiple myeloma. Nature. 2011;471:467-72. 
13. Lohr JG, Stojanov P, Carter SL, et al. Widespread genetic heterogeneity in multiple myeloma: implications for targeted therapy. Cancer Cell. 2014;25:91-101.

•• Massive paralle sequencing analysis of a large cohort of MM patients, focusing on implications for targeted therapy.

14. Bolli N, Avet-Loiseau H, Wedge DC, et al. Heterogeneity of genomic evolution and mutational profiles in multiple myeloma. Nature communications. 2014;5:2997.

-• WES study thoroughly investigating MM mutational profiles and genomic evolution.

15. Walker BA, Boyle EM, Wardell CP, et al. Mutational Spectrum, Copy Number Changes, and Outcome: Results of a Sequencing Study of Patients With Newly Diagnosed Myeloma. Journal of clinical oncology : official journal of the American Society of Clinical Oncology. 2015;33:3911-20.

-. Currently the largest WES study in MM, comprising 463 patients enrolled onto the UK National Cancer Research Institute Myeloma XI trial.

16. Lionetti M, Barbieri M, Todoerti $\mathrm{K}$, et al. Molecular spectrum of BRAF, NRAS and KRAS gene mutations in plasma cell dyscrasias: implication for MEK-ERK pathway activation. Oncotarget. 2015;6:24205-17.

17. Dziembowski A, Lorentzen E, Conti E, et al. A single subunit, Dis3, is essentially responsible for yeast exosome core activity. Nature structural \& molecular biology. $2007 ; 14: 15-22$

18. Tomecki R, Drazkowska K, Kucinski I, et al. Multiple myeloma-associated hDIS3 mutations cause perturbations in cellular RNA metabolism and suggest hDIS3 PIN domain as a potential drug target. Nucleic acids research. 2014;42:1270-90.

19. Lionetti M, Barbieri M, Todoerti K, et al. A compendium of DIS3 mutations and associated transcriptional signatures in plasma cell dyscrasias. Oncotarget. 2015;6:26129-41.

20. Segalla S, Pivetti S, Todoerti K, et al. The ribonuclease DIS3 promotes let-7 miRNA maturation by degrading the pluripotency factor LIN28B mRNA. Nucleic acids research. 2015. 
21. Kuchta K, Muszewska A, Knizewski L, et al. FAM46 proteins are novel eukaryotic noncanonical poly(A) polymerases. Nucleic acids research. 2016;44:3534-48.

22. Boyd KD, Ross FM, Walker BA, et al. Mapping of chromosome $1 \mathrm{p}$ deletions in myeloma identifies FAM46C at $1 \mathrm{p} 12$ and CDKN2C at $1 \mathrm{p} 32.3$ as being genes in regions associated with adverse survival. Clinical cancer research : an official journal of the American Association for Cancer Research. 2011;17:7776-84.

23. Barbieri M, Manzoni M, Fabris S, et al. Compendium of FAM46C gene mutations in plasma cell dyscrasias. British journal of haematology. 2015.

24. Lionetti M, Barbieri M, Manzoni M, et al. Molecular spectrum of TP53 mutations in plasma cell dyscrasias by next generation sequencing: an Italian cohort study and overview of the literature. Oncotarget. 2016.

25. Shaffer AL, Emre NC, Lamy L, et al. IRF4 addiction in multiple myeloma. Nature. 2008;454:226-31.

26. Chen L, Wang S, Zhou Y, et al. Identification of early growth response protein 1 (EGR-1) as a novel target for JUN-induced apoptosis in multiple myeloma. Blood. 2010;115:61-70.

27. Keats JJ, Fonseca R, Chesi M, et al. Promiscuous mutations activate the noncanonical NFkappaB pathway in multiple myeloma. Cancer Cell. 2007;12:131-44.

28. Annunziata CM, Davis RE, Demchenko $\mathrm{Y}$, et al. Frequent engagement of the classical and alternative NF-kappaB pathways by diverse genetic abnormalities in multiple myeloma. Cancer Cell. 2007;12:115-30.

29. Walker BA, Wardell CP, Murison A, et al. APOBEC family mutational signatures are associated with poor prognosis translocations in multiple myeloma. Nature communications. 2015;6:6997.

30. Alexandrov LB, Nik-Zainal S, Wedge DC, et al. Signatures of mutational processes in human cancer. Nature. 2013;500:415-21.

31. Weinhold N, Ashby C, Rasche L, et al. Clonal selection and double-hit events involving tumor suppressor genes underlie relapse in myeloma. Blood. 2016;128:1735-44. 
•• Longitudinal study combining gene expression profiling, copy number arrays and WES on 33 patients to elucidate the mechanisms underlying relapse in MM.

32. Bahlis NJ. Darwinian evolution and tiding clones in multiple myeloma. Blood. 2012;120:9278.

33. Brioli $\mathrm{A}$, Melchor $\mathrm{L}$, Cavo $\mathrm{M}$, et al. The impact of intra-clonal heterogeneity on the treatment of multiple myeloma. British journal of haematology. 2014;165:441-54.

34. Melchor L, Brioli A, Wardell CP, et al. Single-cell genetic analysis reveals the composition of initiating clones and phylogenetic patterns of branching and parallel evolution in myeloma. Leukemia. 2014;28:1705-15.

35. Walker BA, Wardell CP, Melchor L, et al. Intraclonal heterogeneity is a critical early event in the development of myeloma and precedes the development of clinical symptoms. Leukemia. 2014;28:384-90.

36. Keats JJ, Chesi M, Egan JB, et al. Clonal competition with alternating dominance in multiple myeloma. Blood. 2012;120:1067-76.

37. Egan JB, Shi CX, Tembe W, et al. Whole-genome sequencing of multiple myeloma from diagnosis to plasma cell leukemia reveals genomic initiating events, evolution, and clonal tides. Blood. 2012;120:1060-6.

38. Williams MJ, Werner B, Barnes CP, et al. Identification of neutral tumor evolution across cancer types. Nature genetics. 2016;48:238-44.

39. Cifola 1, Lionetti M, Pinatel E, et al. Whole-exome sequencing of primary plasma cell leukemia discloses heterogeneous mutational patterns. Oncotarget. 2015;6:17543-58.

- The first whole-exome sequencing study in PPCL, revealing a highly heterogeneous mutational pattern.

40. Zhan F, Huang Y, Colla S, et al. The molecular classification of multiple myeloma. Blood. 2006;108:2020-8.

41. Mulligan G, Lichter DI, Di Bacco A, et al. Mutation of NRAS but not KRAS significantly reduces myeloma sensitivity to single-agent bortezomib therapy. Blood. 2014;123:632-9. 
42. Andrulis M, Lehners $\mathrm{N}$, Capper $\mathrm{D}$, et al. Targeting the BRAF V600E mutation in multiple myeloma. Cancer discovery. 2013;3:862-9.

-• Paper reporting, for the first time, successful treatment of a BRAF-mutated MM patient with the BRAF inhibitor vemurafenib.

43. Sharman JP, Chmielecki J, Morosini D, et al. Vemurafenib response in 2 patients with posttransplant refractory BRAF V600E-mutated multiple myeloma. Clinical lymphoma, myeloma \& leukemia. 2014;14:e161-3.

44. Heuck CJ, Jethava Y, Khan R, et al. Inhibiting MEK in MAPK pathway-activated myeloma. Leukemia. 2016;30:976-80.

45. Poulikakos PI, Zhang C, Bollag G, et al. RAF inhibitors transactivate RAF dimers and ERK signalling in cells with wild-type BRAF. Nature. 2010;464:427-30.

46. Raab MS, Lehners $\mathrm{N}, \mathrm{Xu}$ J, et al. Spatially divergent clonal evolution in multiple myeloma: overcoming resistance to BRAF inhibition. Blood. 2016;127:2155-7.

47. Rashid NU, Sperling AS, Bolli N, et al. Differential and limited expression of mutant alleles in multiple myeloma. Blood. 2014;124:3110-7.

-• RNA deep sequencing analysis uncovering limited and unbalanced expression of mutant alleles in MM.

48. Avet-Loiseau H. Minimal Residual Disease by Next-Generation Sequencing: Pros and Cons. American Society of Clinical Oncology educational book American Society of Clinical Oncology Meeting. 2016;35:e425-30.

49. Paiva B, Puig N, Garcia-Sanz R, et al. Is this the time to introduce minimal residual disease in multiple myeloma clinical practice? Clinical cancer research : an official journal of the American Association for Cancer Research. 2015;21:2001-8.

50. Mailankody S, Korde N, Lesokhin AM, et al. Minimal residual disease in multiple myeloma: bringing the bench to the bedside. Nature reviews Clinical oncology. 2015;12:286-95.

51. Martinez-Lopez J, Lahuerta JJ, Pepin F, et al. Prognostic value of deep sequencing method for minimal residual disease detection in multiple myeloma. Blood. 2014;123:3073-9. 
52. Ladetto $M$, Bruggemann $M$, Monitillo L, et al. Next-generation sequencing and real-time quantitative PCR for minimal residual disease detection in B-cell disorders. Leukemia. 2014;28:1299-307.

53. Faham M, Zheng J, Moorhead M, et al. Deep-sequencing approach for minimal residual disease detection in acute lymphoblastic leukemia. Blood. 2012;120:5173-80.

54. Logan AC, Zhang B, Narasimhan B, et al. Minimal residual disease quantification using consensus primers and high-throughput IGH sequencing predicts post-transplant relapse in chronic lymphocytic leukemia. Leukemia. 2013;27:1659-65.

55. Drandi D, Kubiczkova-Besse L, Ferrero S, et al. Minimal Residual Disease Detection by Droplet Digital PCR in Multiple Myeloma, Mantle Cell Lymphoma, and Follicular Lymphoma: A Comparison with Real-Time PCR. The Journal of molecular diagnostics : JMD. 2015;17:652-60.

56. Rawstron AC, Paiva B, Stetler-Stevenson M. Assessment of minimal residual disease in myeloma and the need for a consensus approach. Cytometry Part B, Clinical cytometry. 2016;90:21-5.

57. Martinez-Lopez J, Sanchez-Vega B, Barrio S, et al. Analytical and clinical validation of a novel in-house deep sequencing method for minimal residual disease monitoring in a phase II trial for multiple myeloma. Leukemia. 2017.

58. van Dongen JJ, Langerak AW, Bruggemann M, et al. Design and standardization of PCR primers and protocols for detection of clonal immunoglobulin and T-cell receptor gene recombinations in suspect lymphoproliferations: report of the BIOMED-2 Concerted Action BMH4-CT98-3936. Leukemia. 2003;17:2257-317.

59. Kumar S, Paiva B, Anderson KC, et al. International Myeloma Working Group consensus criteria for response and minimal residual disease assessment in multiple myeloma. The Lancet Oncology. 2016;17:e328-46.

60. Horak P, Frohling S, Glimm H. Integrating next-generation sequencing into clinical oncology: strategies, promises and pitfalls. ESMO open. 2016;1:e000094. 
61. Klauschen $\mathrm{F}$, Andreeff $\mathrm{M}$, Keilholz $\mathrm{U}$, et al. The combinatorial complexity of cancer precision medicine. Oncoscience. 2014;1:504-9.

62. Berry DA. Adaptive clinical trials in oncology. Nature reviews Clinical oncology. 2011;9:199207.

63. Schork NJ. Personalized medicine: Time for one-person trials. Nature. 2015;520:609-11.

64. Collette L, Tombal B. N-of-1 trials in oncology. The Lancet Oncology. 2015;16:885-6.

65. Gucalp A, Epstein AS, Seidman AD, et al. Piloting IBM Watson Oncology within Memorial Sloan Kettering's regional network [abstract]. Journal of Clinical Oncology. 2014;32:e17653.

66. Gucalp A, Epstein AS, Seidman AD, et al. Assessing the performance of Watson for oncology, a decision support system, using actual contemporary clinical cases [abstract]. Journal of Clinical Oncology. 2015;33:8023.

67. Kortum KM, Langer $\mathrm{C}$, Monge $\mathrm{J}$, et al. Targeted sequencing using a 47 gene multiple myeloma mutation panel $(\mathrm{M}(3) \mathrm{P})$ in $-17 \mathrm{p}$ high risk disease. British journal of haematology. 2015;168:507-10.

68. Kortuem KM, Braggio E, Bruins L, et al. Panel sequencing for clinically oriented variant screening and copy number detection in 142 untreated multiple myeloma patients. Blood Cancer J. 2016;6:e397.

69. Kortum KM, Mai EK, Hanafiah $\mathrm{NH}$, et al. Targeted sequencing of refractory myeloma reveals a high incidence of mutations in CRBN and Ras pathway genes. Blood. 2016;128.1226-33.

70. Bolli N, Li Y, Sathiaseelan V, et al. A DNA target-enrichment approach to detect mutations, copy number changes and immunoglobulin translocations in multiple myeloma. Blood Cancer J. 2016;6:e467.

71. Jimenez C, Jara-Acevedo M, Corchete LA, et al. A Next-Generation Sequencing Strategy for Evaluating the Most Common Genetic Abnormalities in Multiple Myeloma. The Journal of molecular diagnostics : JMD. 2017;19:99-106. 
72. Lohr JG, Kim S, Gould J, et al. Genetic interrogation of circulating multiple myeloma cells at single-cell resolution. Science translational medicine. 2016;8:363ra147.

-• Pivotal study suggesting the potential of MM circulating tumor cells analysis to replace BM biopsy.

73. Manier S, Park J, Freeman S, et al. Whole-Exome Sequencing and Targeted Deep Sequencing of cfDNA Enables a Comprehensive Mutational Profiling of Multiple Myeloma [abstract]. Blood. 2016;128:197.

74. Mithraprabhu S, Khong T, Ramachandran M, et al. Circulating tumour DNA analysis demonstrates spatial mutational heterogeneity that coincides with disease relapse in myeloma. Leukemia. 2017.

75. Mitra AK, Mukherjee UK, Harding T, et al. Single-cell analysis of targeted transcriptome predicts drug sensitivity of single cells within human myeloma tumors. Leukemia. 2016;30:1094-102.

76. Adamia S, Reiman T, Crainie M, et al. Intronic splicing of hyaluronan synthase 1 (HAS1): a biologically relevant indicator of poor outcome in multiple myeloma. Blood. 2005;105:483644.

77. Nagoshi H, Taki T, Chinen Y, et al. Transcriptional dysregulation of the deleted in colorectal carcinoma gene in multiple myeloma and monoclonal gammopathy of undetermined significance, Genes, chromosomes \& cancer. 2015;54:788-95.

78. Rashid N, Minvielle S, Magrangeas F, et al. Alternative Splicing Is a Frequent Event and Impacts Clinical Outcome in Myeloma: A Large RNA-Seq Data Analysis of NewlyDiagnosed Myeloma Patients [abstract]. Blood. 2014;124:638.

79. Amodio N, Di Martino MT, Neri A, et al. Non-coding RNA: a novel opportunity for the personalized treatment of multiple myeloma. Expert opinion on biological therapy. 2013;13 Suppl 1:S125-37.

80. Nobili L, Lionetti M, Neri A. Long non-coding RNAs in normal and malignant hematopoiesis. Oncotarget. 2016;7:50666-81. 
81. Amodio N, D'Aquila P, Passarino G, et al. Epigenetic modifications in multiple myeloma: recent advances on the role of DNA and histone methylation. Expert opinion on therapeutic targets. 2017;21:91-101.

82. Pan $\mathrm{H}$, Jiang $\mathrm{Y}$, Boi $\mathrm{M}$, et al. Epigenomic evolution in diffuse large B-cell lymphomas. Nature communications. 2015;6:6921. 
Figure 1. Most significantly mutated genes in MM. Frequencies of coding nonsynonymous mutations observed in each of the three main WGS/WES studies in MM $[13,14,15]$ are reported for genes having an average mutation rate of at least $5 \%$ and reaching statistical significance in at least two studies.

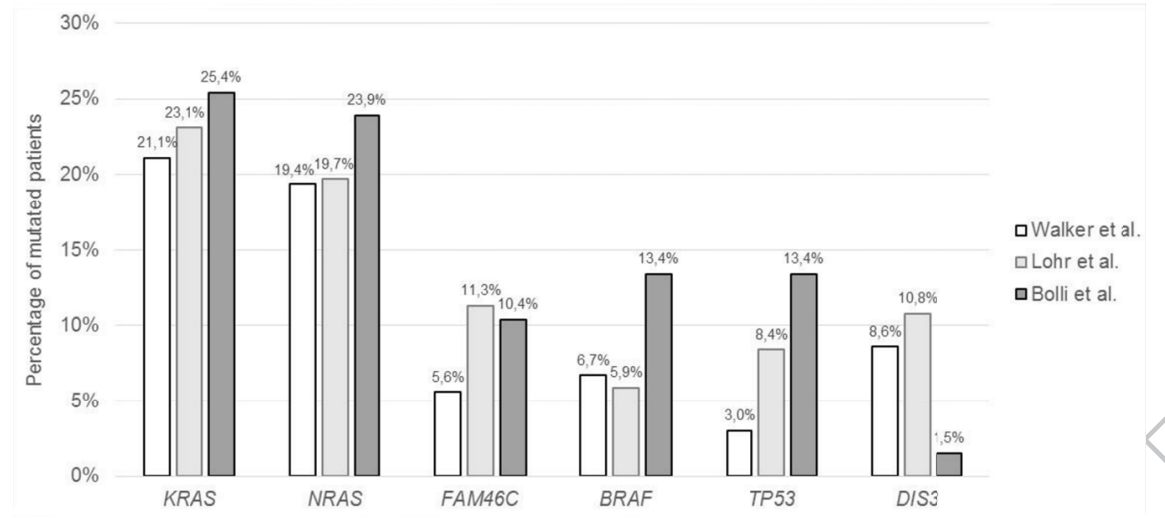


Figure 2. Summary of $B R A F$ mutations in primary $M M / P C L$ patients $[13,14,15,16]$. The three conserved regions (CR) in all RAF proteins (CR1, CR2 and CR3) are shown, with the activation segment within CR3 indicated by diagonal lines. The number of patients (vertical axis) carrying missense and indel somatic mutations affecting the indicated amino acid residues is represented.

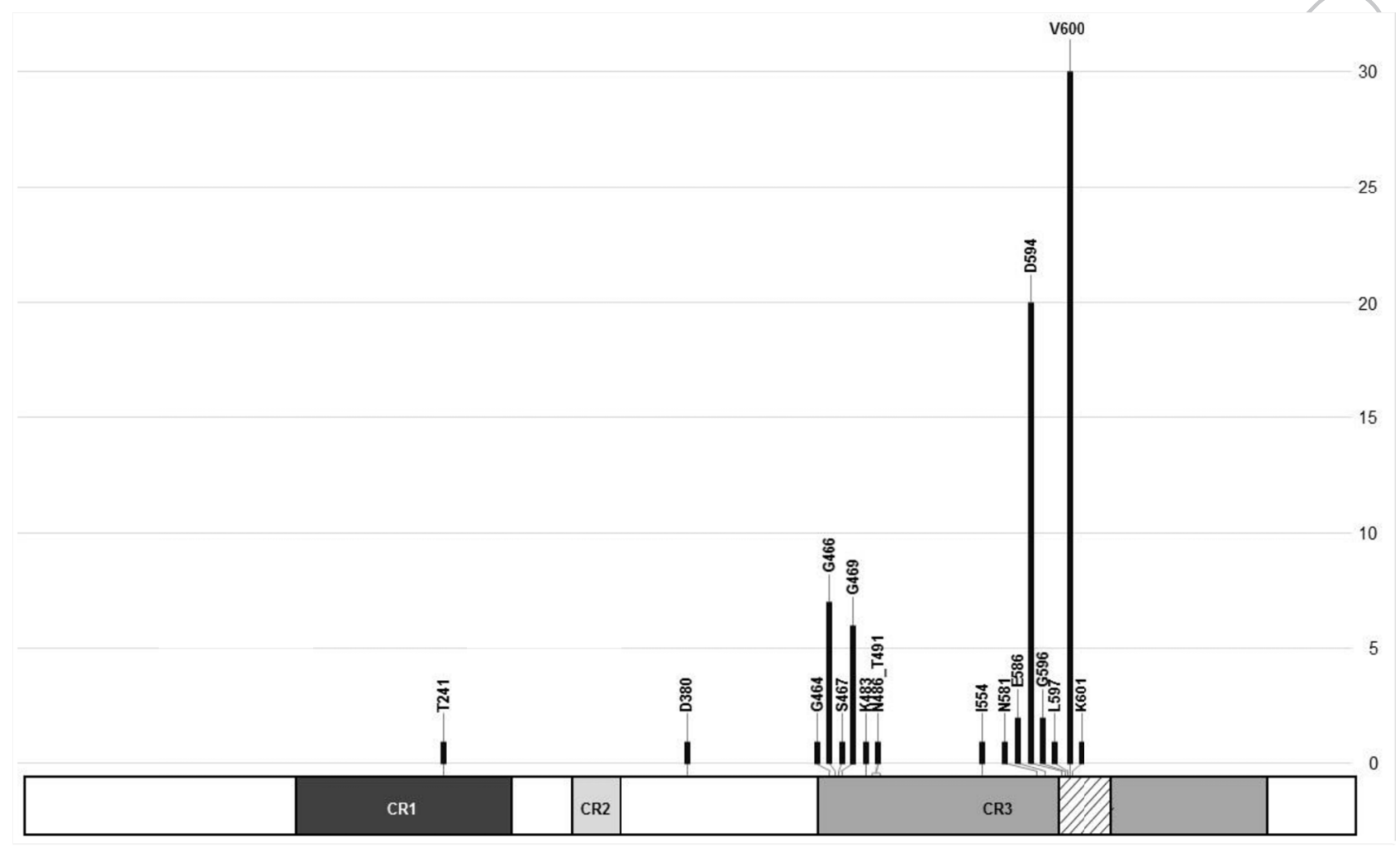


Figure 3. Outline of missense, nonsense and indel mutations in DIS3 [13, 14, 15, 19] (A) and FAM46C [13, 14, 15, 23] (B) genes. The number of patients (vertical axis) carrying somatic mutations affecting the indicated amino acid residues is represented.

A

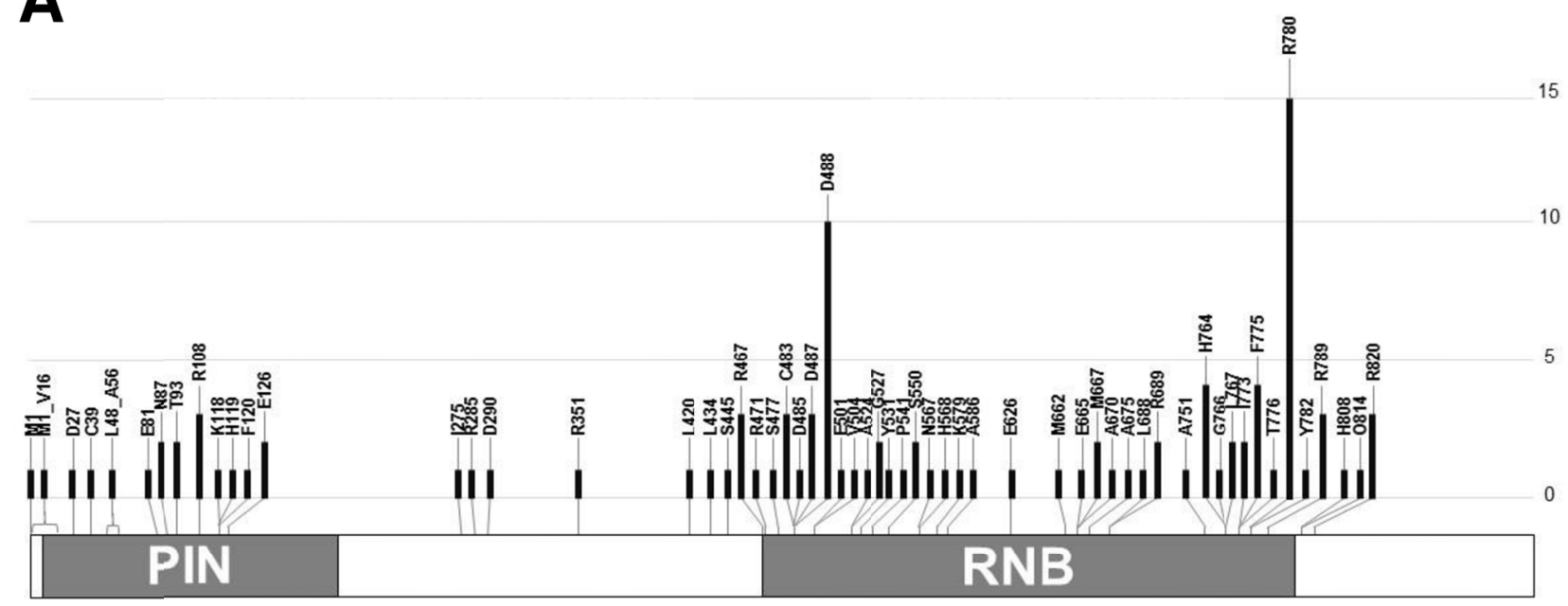

B

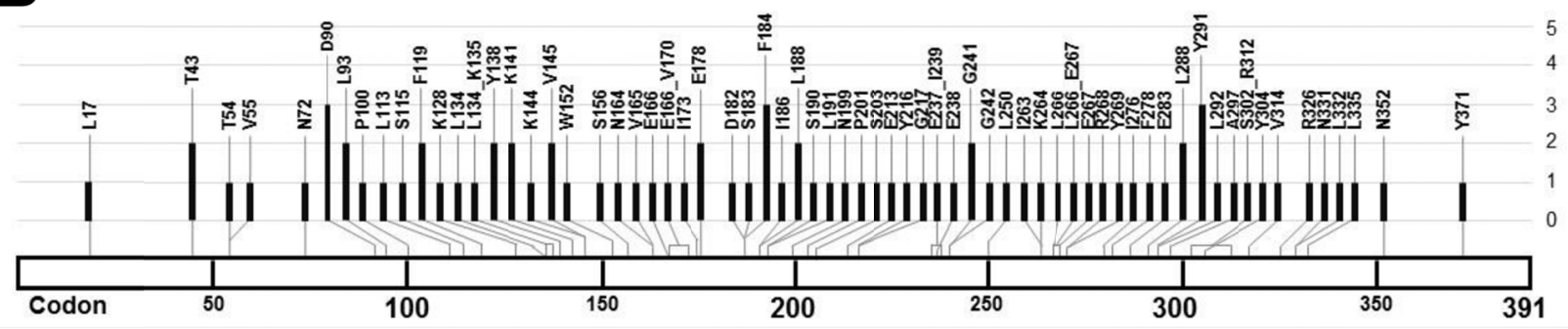


Figure 4. Representation of TP53 mutations identified in PC dyscrasia patients. Amino acid affected by missense, nonsense or indel somatic mutations are indicated on the scheme of the structural organization of TP53 protein, in correspondence of a vertical bar proportional in length to the number of mutated patients (vertical axis) $[13,14,15,24]$.

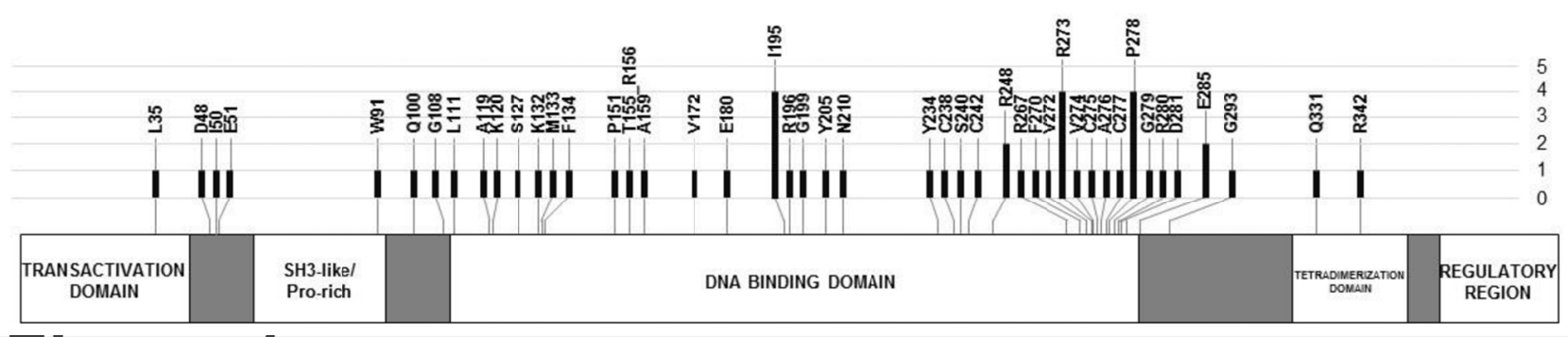


Table 1. Genes affected by druggable mutations in MM.

\begin{tabular}{ccc}
\hline Gene & $\begin{array}{c}\text { Percentage of mutated MM } \\
\text { patients }\end{array}$ & Targeted therapy \\
\hline KRAS & $23.2 \%$ & MEK inhibitor \\
NRAS & $21 \%$ & MEK inhibitor \\
BRAF & $8.7 \%$ & Vemurafenib \\
ROS1 & $3.1 \%$ & Foretinib \\
CCND1 & $2.7 \%$ & Pablociclib \\
FGFR3 & $1.5 \%$ & Masitinib \\
MLL & $1.2 \%$ & EPZ-5676 \\
PIK3CA & $1.2 \%$ & GDC-0941 \\
FGFR2 & $1.2 \%$ & Masitinib \\
FLT3 & $1 \%$ & Sunitinib
\end{tabular}

Genes affected by druggable mutations in MM according to what reported by Walker et al. [15] and found mutated in at least $1 \%$ of patients when averaging the frequencies reported by Walker et al. [15],Bolli et al. [14], and Lohr et al. [13]. 